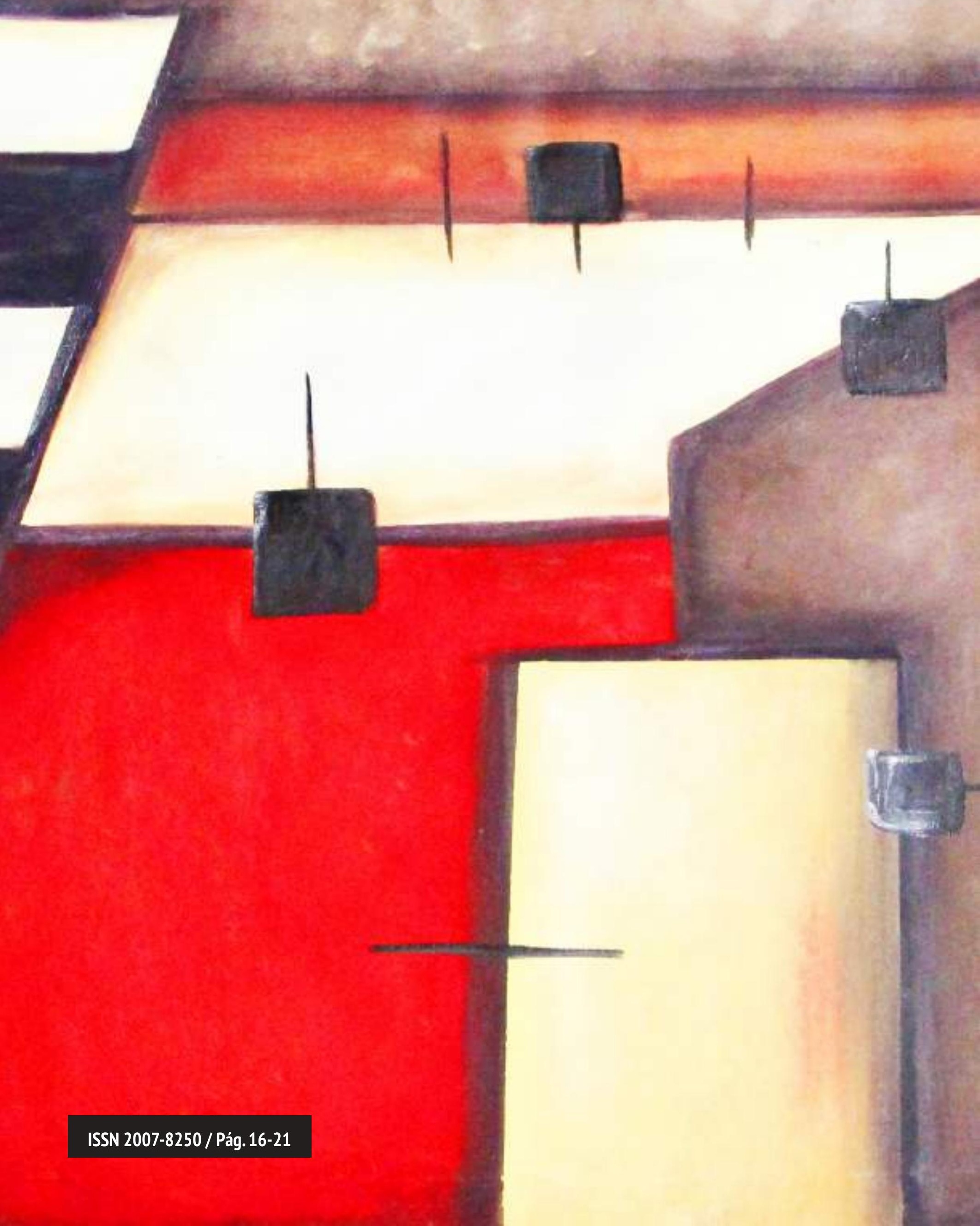




\section{REFLEXIONES EN TORNO \\ A LOS ESTUDIOS DE POSTGRADO \\ DE LA UNIVERSIDAD AUTÓNOMA \\ DE NUEVO LEÓN}

\section{Libertad Leal Lozano}

No. 3 - Diciembre 1990

ficiencia, productividad y calidad son aspectos que exigen las empresas 1 en la selección de su personal. Hoy día es más difícil preparar profesionales adecuados al mercado de trabajo de sociedades cada día más avanzadas tecnológicamente. A esto se puede externar una serie de razones, entre ellas, destacamos las siguientes: La educación superior desvinculada de la realidad, la ineficiencia de los sistemas educativos, o la inadecuación de los perfiles profesionales al mercado laboral. 
$\mathrm{N}$ aturalmente en el fondo de todo esto se encuentran pocos estudios de mercado hechos dentro de las mismas organizaciones educativas a diferencia de los corporativos donde gastan y procuran mantener al día su personal en todos los niveles. Es necesario que esto lo haga gente calificada que además de conocer el sistema hacia adentro tengan experiencias de la problemática de inserción laboral sin embargo es indiscutible que el proceso de selección en las empresas tiende a ser mucho más actualizado 0 al menos está a la par en función de sus propios intereses.

En cambio, las universidades cada día se distancian más del sector productivo por las siguientes razones:

- El mantener carreras que ya no responden a los requerimientos del mercado.

- Crear segmentos de profesionales dirigidos a problemáticas de algunas sociedades muy desarrolladas pero pocos para el tipo de sociedad que tenemos.

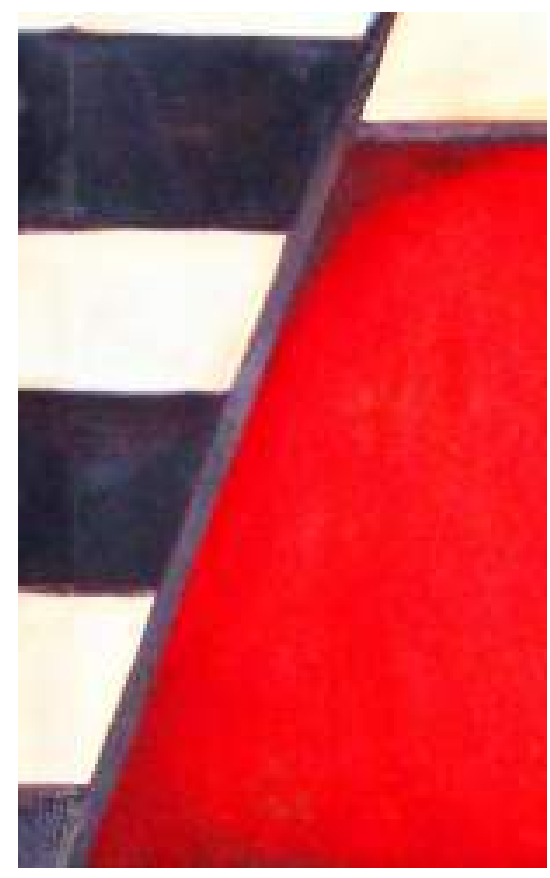

\section{Las organizaciones cambian sus estructuras, sus formas de lidiar con los problemas y sobre todo sus formas de ajustarse al gran cambio social de nuestra época}

Quizá por eso ahora se dé la importancia al nivel técnico-profesional.

En este sentido un concepto que nos parece neurálgico en todo, el proceso de explicaciones de fenómenos anteriormente mencionados es el de organizaciones. Las organizaciones cambian sus estructuras, sus formas de lidiar con los problemas y sobre todo sus formas de ajustarse al gran cambio social de nuestra época.

Lo que nos parece imposible de entender es cómo una organización como la universidad, que cuenta con teóricos y visionarios dentro de sí misma en muchas áreas del conoci-

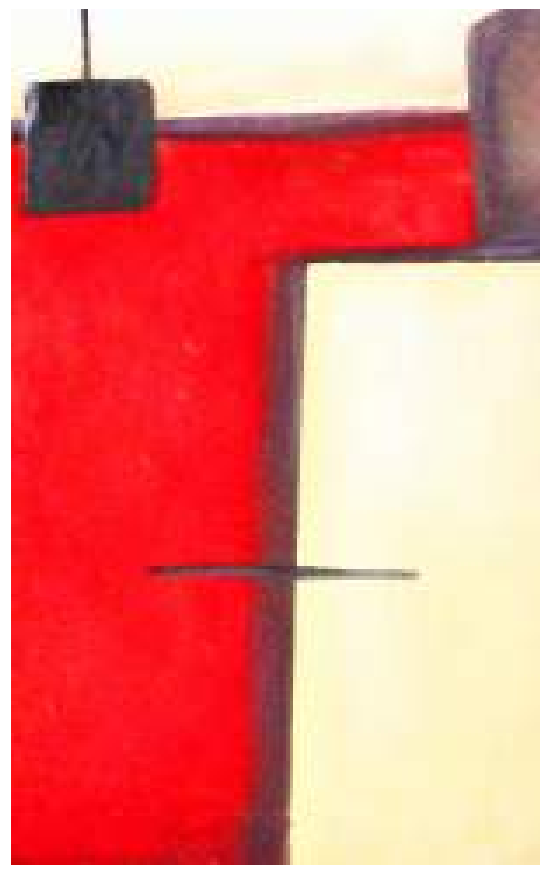

miento, no pueda cambiar su propia organización tan sensible dado que es un sistema abierto y complejo que afecta y es afectado por el entorno social. La idea de comprar estas organizaciones tanto educativas como empresariales es tratar de entender sus problemas de entrada en el proceso de cambio de una organización.

Para eso consideramos un sólo aspecto de toda esta estructura; la organización del Postgrado. El Postgrado debe ser la parte más actualizada donde incidan los mejores recursos humanos en términos de calidad institucional, pero las cifras nos arrojan lo contrario.

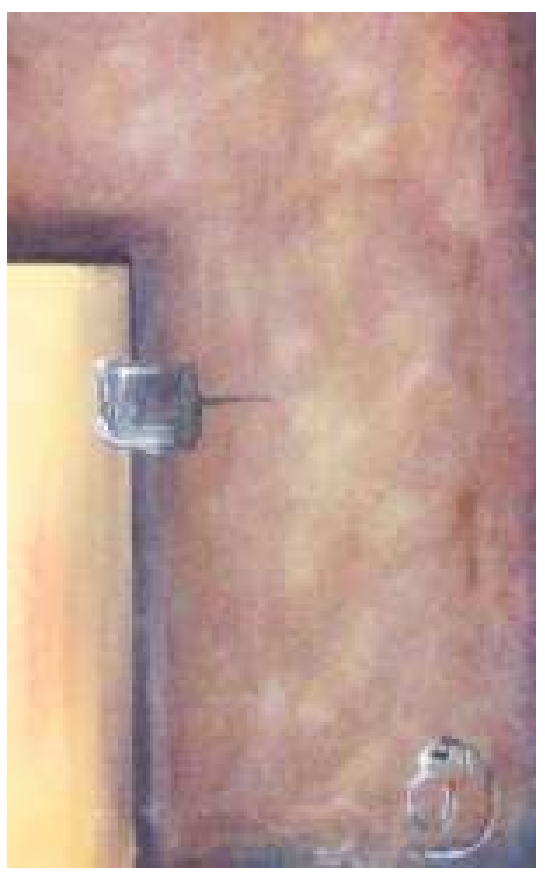




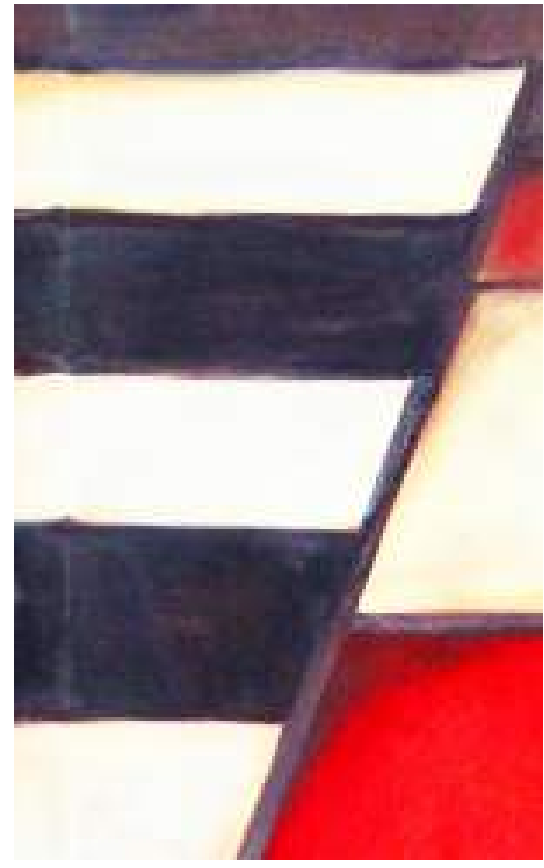

Cuando en otros países, el Postgrado sucede como una vertiente del proceso educativo, y donde están los mejores profesionales, aquí en nuestro país, el Postgrado se ha reducido sólo a un apéndice de la institución, donde se aglomeran, los políticos, y muy pocos académicos. Por ejemplo el uso de los cursos de opción a título, manifiesta una baja calidad del Postgrado porque de alguna manera dinamizan a la Institución en función de sus propias necesidades, sacar el título y sólo eso.

Otro elemento que debe ser connatural es el fortalecimiento del Postgrado a través de la investigación,
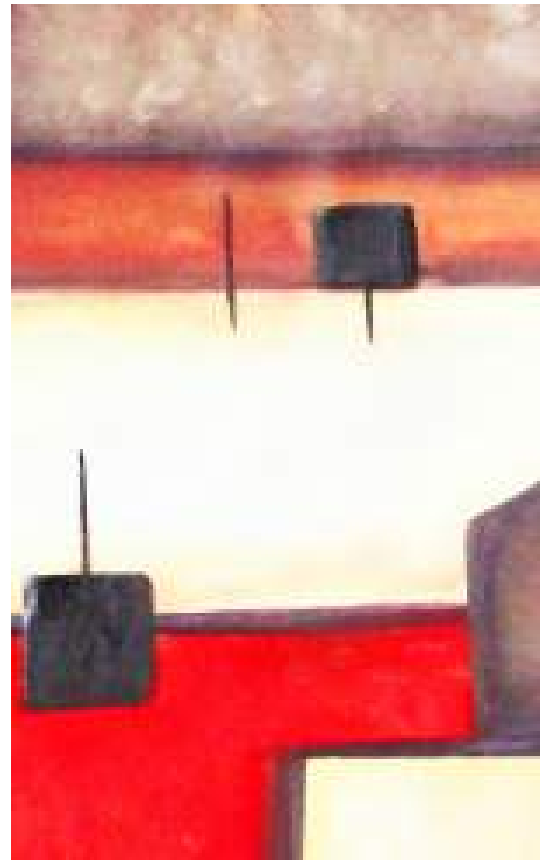

pero por lo regular encontramos que poco o nada tiene que ver la investigación con el Postgrado, esto lleva a concebir a la misma investigación como un arma a los fines estrictamente docentes de la Universidad.

Mucho de lo anterior es sumamente difícil de controlar, ya que no existen órganos reguladores y cuidadores de la excelencia lo que ha hecho del Postgrado un sistema anárquico de la Institución. De esta manera encontramos programas de postgrado con alto nivel de exigencia y al mismo tiempo programas de fin de semana. El mismo reclutamiento de recursos humanos en éste tipo de organiza-
En nuestro país, el Postgrado se ha reducido sólo a un apéndice de la institución donde se aglomeran los políticos y muy pocos académicos

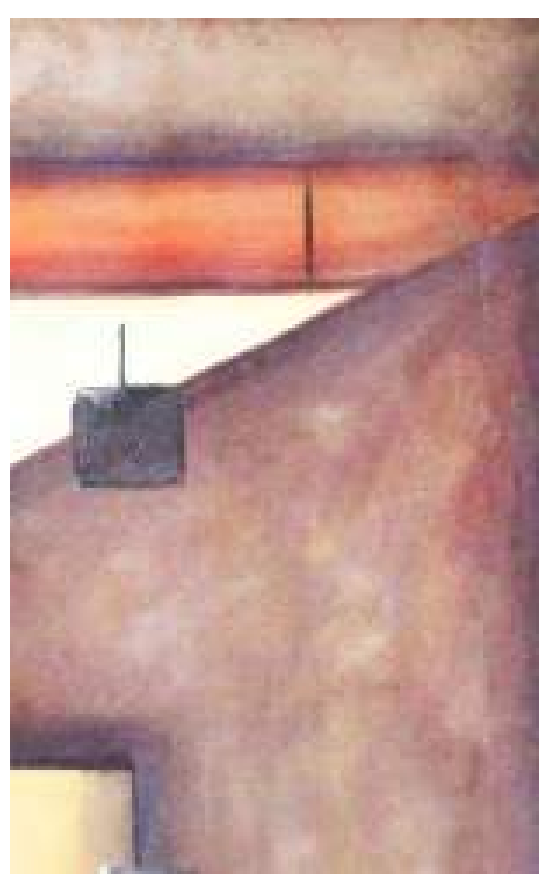

ción universitaria no es tan selectivo como en las organizaciones empresariales, donde les preocupa qué tipo de profesionales van a incorporar, en cambio en el sistema universitario se reclutan elementos que saquen la hora de clase y resuelvan el problema de inmediato en su mayoría.

Problemas latentes en el Postgrado:

- La infraestructura reducida en cuando al acervo bibliográfico, espacios de trabajo, laboratorios y sobre todo herramientas como computadoras, microscopios, etc.

- El Postgrado reducido a un proceso de enseñanza y no como producto de la investigación.

- Falta de asesores disponibles que orienten las investigaciones de los estudiantes de Postgrado.

- Poca relación inter y multidisciplinaria en programas institucionales.

- No existen órganos de disfunción internos del Postgrado y de la investigación que mantengan actualizados a los estudiantes y maestros en términos generales. 


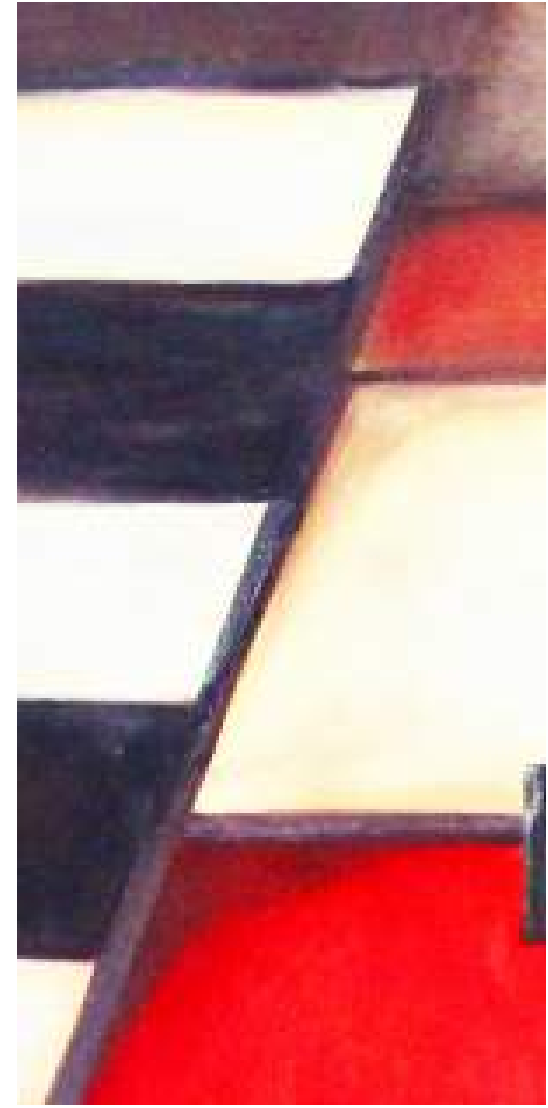

- Los estudios de Postgrado y los cursos de actualización no se consideran de manera escalafonaria en el profesional universitario.

- Abrir los cursos de Postgrado a toda la Universidad con valor currícular a fin de que los maestros se mantengan actualizados.

- Pugnar porque el Postgrado tenga presencia legal ante el Consejo Universitario y dejen de decidir por él.

- Elaborar un manual del estudiante de Postgrado que contenga todos los aspectos de carácter Académico, Administrativos y que, incluso, defina de manera clara la Especialización, la Maestría y el Doctorado ya que el actual Modelo de Desarrollo de Postgrado de la Universidad Autónoma de Nuevo León, es muy ambiguo.

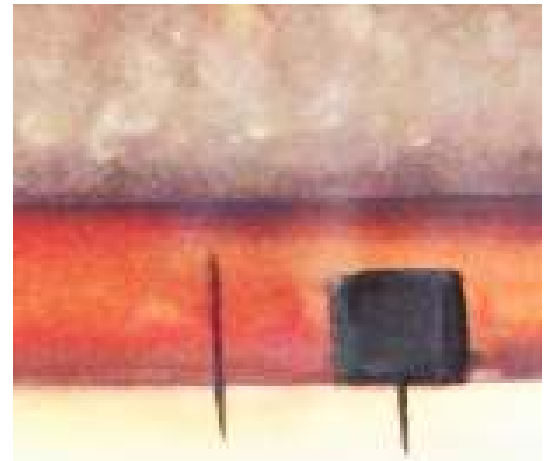

Preocupados por estar en primera línea consideramos que el Postgrado es "la niña de los ojos" de la Universidad, es ahí donde se puede medir la calidad de trabajo que la Universidad tiene en cuanto a mantenerse a tono con los nuevos modelos de la educación, por lo que sugerimos lo siguiente:

1. Desarrollo un modelo donde la parte de investigación del Postgrado tome la primera posición en el desarrollo de la función universitaria.

2. Buscar los mecanismos más adecuados para darle a los investigadores y docentes del Postgrado su ubicación en el programa general de la universidad.

3. Eliminar de los Postgrados la repetición de curso (básicos) dejando sólo algunos de ellos como Cátedras, donde puedan acudir los estudiantes independientemente de su Facultad.

4. Crear cátedras para los Maestros que se han mantenido por un buen número de años enseñando con alto nivel de eficiencia dichos cursos.

5. Fundir programas de Postgrado con el fin de optimizar los recursos: Maestrías en Biología, Medicina, Agronomía y Veterinaria, por ejemplo, para que cada estudiante pueda diseñar su cu-
El Postgrado es donde se puede medir la calidad de trabajo que la Universidad tiene en cuanto a mantenerse a tono con los nuevos modelos de la educación 


\section{Nosotros creemos en la calidad de nuestro recurso humano, sólo falta reorientar nuestros esfuerzos}

rrículum de acuerdo a intereses y aspiraciones personales.

6. Pagar al profesional del Postgrado su tiempo en función de los honorarios profesionales pagados en el mercado extrauniversitario.

7. Establecer intercambios donde un curso que se tome en otras instituciones tenga validez dentro de la Universidad.

8. Descontaminar el Postgrado del control de la licenciatura, en cuanto a su administración.

9. Pagar tiempo completo y exclusivo a quien trabaje con la organización del Postgrado (si se quiere mejorarlo).

10. Ofrecer entrenamiento a estudiantes y maestros en otras instituciones de Educación Superior en áreas de conocimiento de frontera.

11. Programar menos cursos y mayor trabajo de investigación a fin de que la tesis sea resultado de un trabajo prolongado del Postgrado y no sólo un trabajo para salir del paso.

Por último, sólo resta señalar que la Educación a nivel de Postgrado es una necesidad que urge priorizar. Cada día las fuentes de trabajo exigen mayor especialización, conforme la tecnología avanzada. No es posible que una institución como la nuestra se entretenga en lo inmediato cuando el futuro este encima. Nosotros creemos en la calidad de nuestro recurso humano, sólo falta reorientar nuestros esfuerzos en pro de una eficiencia y productividad más allá de lo meramente burocrático.

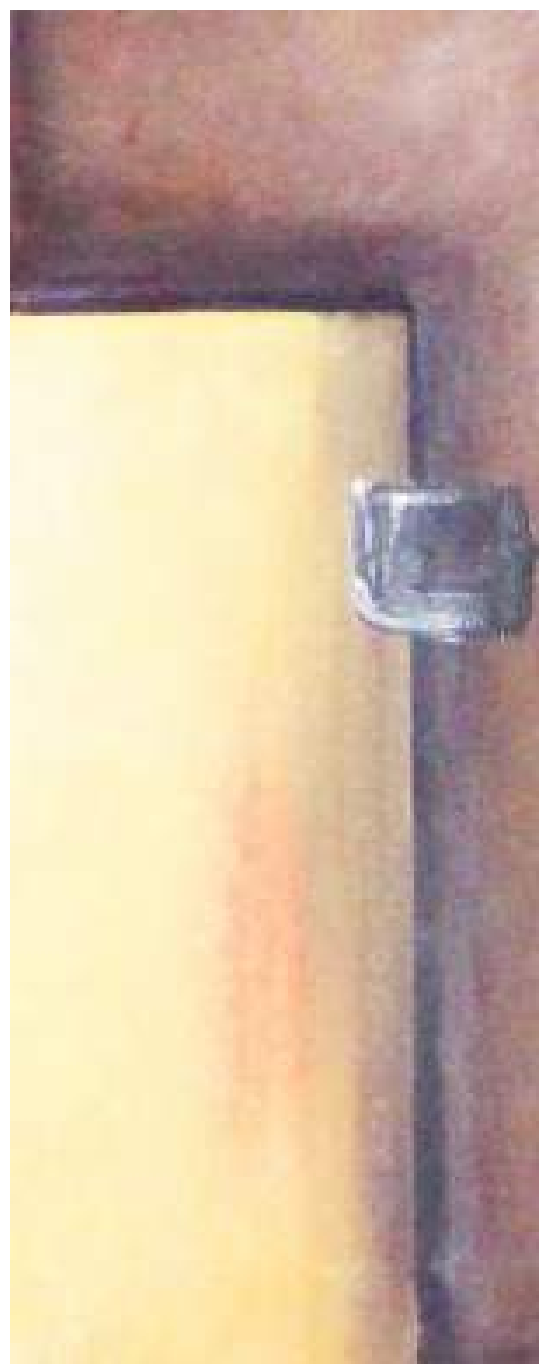

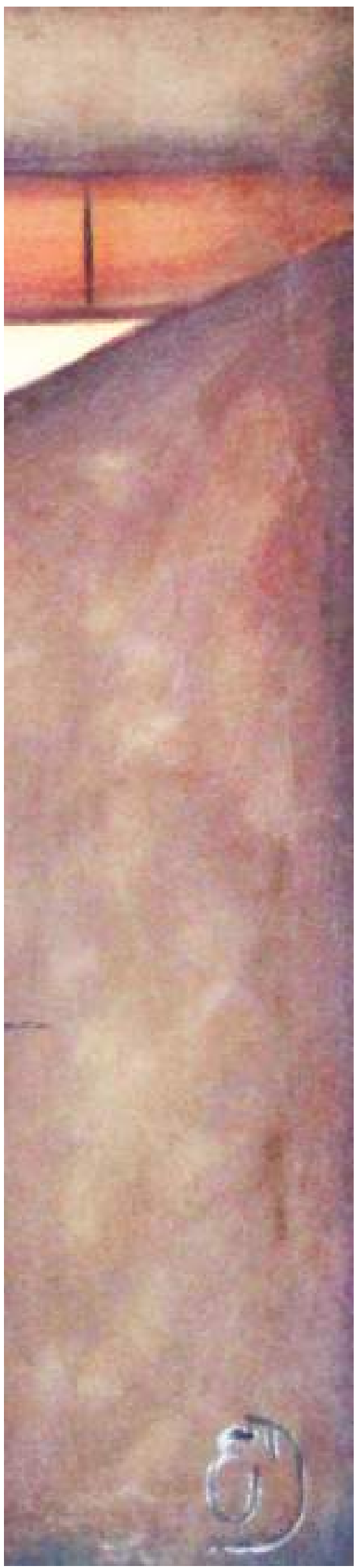

